\title{
Internalizing Disorders as Risk Factors for Early School Leaving: A Systematic Review
}

\author{
Ole Melkevik $^{1}$ (1) $\cdot$ Wendy Nilsen $^{2} \cdot$ Miriam Evensen $^{2} \cdot$ Anne Reneflot $^{2} \cdot$ \\ Arnstein Mykletun ${ }^{2,3,4,5}$
}

Received: 25 January 2016/ Accepted: 9 February 2016/Published online: 23 February 2016

(C) Springer International Publishing 2016

\begin{abstract}
Internalizing problems (anxiety and depression) are repeatedly reported to be associated with dropout from secondary school and adverse social and health outcomes later in life, but the evidence appears inconsistent. The aim of this study was to systematically review the evidence for internalizing problems as a risk factor for early school leaving. A systematic search in major databases with thematic search strings for both internalizing disorders and early school leaving yielded 3188 records, of which 14 were included after screening and reading of full texts by two researchers independently after preset inclusion and exclusion criteria. The review process identified four prospective, three trajectory and seven retrospective studies. Variation in measurements, design, adjustments
\end{abstract}

Ole Melkevik

ome@arbejdsmiljoforskning.dk

Wendy Nilsen

wendy.nilsen@afi.hioa.no

Miriam Evensen

miriam.evensen@fhi.no

Anne Reneflot

anne.reneflot@fhi.no

Arnstein Mykletun

arnstein.mykletun@fhi.no

1 National Rersearch Centre for the Working Environment, Lerso Parkalle 105, 2100 Copenhagen O, Denmark

2 The Norwegian Institute of Public Health, Postboks 4404, Nydalen, 0403 Oslo, Norway

3 Department of Community Medicine, University of Troms $\emptyset$, Troms $\varnothing$, Norway

4 Center for Work and Mental Health, Nordland Hospital Trust, Bodø, Norway

5 University of New South Wales, Sydney, Australia and statistics precluded meta-analyses. The results were mixed. Internalizing problems were reported to increase the risk of early school leaving in prospective studies, but not when adjusted for conduct disorder and ADHD. It is somewhat unclear if the association is robust for adjustment for externalizing problems in studies applying trajectory analyses and non-diagnostic instruments. There were no systematic gender differences in reported associations. In conclusion, internalizing problems were repeatedly reported to increase the risk of early school leaving, but this association was partly or fully accounted for by adjustment for comorbid externalizing problems. However, whether this is adjusting for mediating or confounding factors remains unanswered.

Keywords Adolescence · Internalizing - Anxiety · Depression · Dropout · Early School Leaving

\section{Introduction}

Early school leaving is associated with adverse outcomes for individuals in terms of social integration, economic prospects and future health (Fryers et al. 2003). In turn, it is also costly for the welfare state partly due to increased future welfare dependency among adults with lower levels of educational attainment (Nevala et al. 2011). In Europe, $14.4 \%$ have not completed upper secondary school within the age of 24 (Nevala et al. 2011), and $25.1 \%$ of young adults in the US have not attained their diploma within 4 years of entering public high school (Lee et al. 2011). Better understanding of mechanisms causing early school leaving may inform tailored interventions (Nevala et al. 2011; OECD 2011).

Adverse outcomes of internalizing disorders including anxiety and depression are well documented. For example, 
depression is a risk factor for increased mortality across various causes of death (Mykletun et al. 2007), and the association is in strength comparable to that of smoking and mortality (Mykletun et al. 2009). Both anxiety and depression are strong predictors of later sickness absence (Knudsen et al. 2013) and disability benefit award (Mykletun et al. 2006). The associations between internalizing problems and such adverse outcomes are found to be stronger in younger individuals than in older individuals (Mykletun et al. 2006, 2007). Both depression and anxiety contribute substantially to the global burden of disease (Whiteford et al. 2013). On this background, it is therefore a compelling hypothesis that early school leaving may to some extent be a consequence of anxiety and depression.

Some large and well known studies have supported the hypothesis that early onset psychiatric disorders are causally associated with early school leaving. For instance, Kessler et al. (1995) reported that individuals with psychiatric disorders account for about $14.2 \%$ of the high school dropout population in the US. Vander Stoep et al. (2003) report that about $46 \%$ of high school non-completion in the US is likely to be attributed to psychiatric disorders. The proportion of early school leaving attributed specifically to internalizing disorders is not known, but the relatively high prevalence of these disorders, and their substantial comorbidity with other mental disorders (Kessler et al. 2005b) warrants a thorough investigation of the role of internalizing disorders as potential risk factors for early school leaving. Beyond these well-known studies, findings are more mixed, including studies reporting no association between early school leaving and internalizing disorders (Miech et al. 1999), and some even negative associations (Borges et al. 2011). Importantly, the extent to which early onset internalizing disorders are associated with early school leaving feeds into the discussion of whether the frequently reported educational gradient in common mental disorders (Bjelland et al. 2008; Wang et al. 2010) can at least partially be attributed to impaired social mobility caused by early onset anxiety and depression.

A challenge in identifying the association between internalizing disorders and early school leaving is to determine the extent to which this association is partly or fully attributed to confounders such as social inequality, family characteristics, school related factors, or comorbidity. Comorbidity between externalizing and internalizing problems is well recognized (Kessler et al. 2005b). It is therefore, important to assess the extent to which associations between internalizing problems and early school leaving are consistent when accounting for comorbidity. Evidence suggesting inconsistent associations between individuals with and without comorbid disorders may potentially suggest biased estimates in studies that do not account for comorbidity. The investigation of the importance of comorbidity is particularly important in light of the well-established evidence for associations between externalizing problems and later early school leaving (Kessler et al. 1995; Miech et al. 1999; Esch et al. 2014). Systematic reviews have indicated that externalizing behavioral problems such as ADHD (van der Kolk et al. 2011; Esch et al. 2014) and cannabis use (Macleod et al. 2004; Esch et al. 2014) are important risk factors for early school leaving, while mood and anxiety disorders have been found to be relatively weakly associated with early school leaving (Esch et al. 2014).

Gender differences have been well established in the prevalence rates of both internalizing problems and early school leaving. While girls are found to be more likely to suffer internalizing problems (Kessler et al. 2005a), boys have been found to have higher prevalence rates of early school leaving (Nevala et al. 2011). Analyses of gender differences in the associations between internalizing problems and early school leaving does, however, appear to be inconsistent across studies as Fergusson and Woodward (2002) found no significant gender differences whereas McLeod and Fettes found lower risk of early school leaving for girls with high anxiety symptoms during adolescence (2007). This study will, therefore, attempt to clarify whether the cumulative body of evidence supports consistent associations across gender or a gender modification of the association between internalizing problems and early school leaving.

\section{Current Study}

While Esch et al. (2014) have recently published a comprehensive systematic review investigating the associations between a broad spectrum of mental disorders and early school leaving, we aim to conduct a more specific review, investigating the extent to which early school leaving may be a consequence of early onset internalizing disorders or symptoms thereof. Furthermore, we aim to focus on research design and residual confounding. As internalizing problems often co-occur with externalizing problems (Kessler et al. 2005b), we will particularly examine to which extent externalizing problems (ADHD), conduct disorder and symptoms thereof may be accounting for the alleged association between internalizing problems and later early school leaving. We will also examine if there is any evidence of gender interaction in this hypothesized association, which is relevant in light of higher prevalence of internalizing problems in girls (Kessler et al. 1993), though lower rates of early school leaving (Nevala et al. 2011). 


\section{Methods}

\section{Search Strategy}

The current study searched ISI web of knowledge, Embase, Medline, PsychInfo, and Scopus (November 30, 2012). No date restrictions were set on the search. Two thematic search strings were used to find articles on the topics of internalizing disorders and early school leaving (see "Appendix"). Additional records where identified by forward search and by searching through reference lists of included articles.

\section{Eligibility Criteria}

The following criteria were used to assess whether studies were suitable for inclusion in the current review:

1. A title referring to relevant topics such as early school leaving or mental disorders/symptoms.

2. An independent variable measuring internalizing problems either as symptoms or as clinical diagnosis indicating anxiety and/or depression.

3. A dichotomous dependent variable indicating whether participants had completed high school/upper secondary school. Studies using educational attainment as a continuous variable were not included.

4. Reported statistical test(s) for differences in risk for early school leaving between either symptom level or diagnostic groups (diagnosis vs. no diagnosis) assessed in childhood or adolescence.

5. A general population sample. Samples that were preselected on particular characteristics such as chronic illness, traumatic experiences, or children with ADHD, externalizing or internalizing behavioral problems were excluded.

\section{Results}

\section{Study Extraction}

Figure 1 shows the flow chart based upon the suggestions of the Prisma group (Moher et al. 2009) for the selection of included studies. The search yielded a total of 3188 records that were first screened by the first author. Papers were excluded based on the relevance of their titles. Second, the abstracts of the 571 remaining records were screened by two researchers (O. M. and W. N.), excluding 428 studies that did not explicitly address topics relevant to early school leaving and mental disorders/symptoms according to eligibility criterion 1 . Next, full text studies were assessed for eligibility according to eligibility criteria 2-5 (by O. M. and W. N.). Reasons for excluding the 129 studies are listed in Fig. 1. At any stage, differences in opinion between the investigators were settled after discussion.

Further, we searched the reference lists of the included records and did forward searches to find more recent studies having cited these. Three studies (Borges et al. 2011; Jayakody et al. 1998; Lee et al. 2009) were found to be relevant for the review, but one study (Jayakody et al. 1998) used a subsample of the data from The National Comorbidity study (NCS) which was also used in Kessler and colleagues (Kessler et al. 1995). In this case, we chose to describe the study of Kessler (Kessler et al. 1995) as both genders were included in the analysis. After searching the reference lists and performing forward searches, we identified two more relevant studies (Lee et al. 2009; Myer et al. 2009). When two or more studies used subsets of the same data, we chose to describe the most recent and/or comprehensive of these studies (Fletcher 2010; McLeod and Fettes 2007), leaving three studies out of the analysis (Fletcher 2008; Needham 2009; McLeod and Kaiser 2004).

\section{Characteristics of Included Studies}

The final sample included 14 studies which are described in Table 1 . The majority of the studies $(\mathrm{n}=11)$ were conducted in USA, Canada, Australia and New Zealand, while one study was done in South Africa (Myer et al. 2009), one in Mexico (Borges et al. 2011), and one study included samples from 16 different countries (Lee et al. 2009). In total, the 14 papers examine 1,14,726 individuals, and were published between 1995 and 2012. The studies are grouped into three categories based on their study designs: (a) prospective variable-oriented studies ( $\mathrm{n}=4$ studies), (b) retrospective studies ( $\mathrm{n}=7$ studies), and (c) trajectory studies ( $\mathrm{n}=3$ studies).

There was considerable variation in measurement of key variables. Two studies (Duchesne et al. 2008; Morin et al. 2011) attained educational status from registries, and the remaining studies collected information about education from either interviews or self-report questionnaires. In the latter alternative, attrition is an obvious limitation, presumably strongly related to outcome variable itself. Internalizing problems were in ten studies based on clinical interviews (Kessler et al. 1995; Borges et al. 2011; Lee et al. 2009; Myer et al. 2009; Miech et al. 1999; Vander Stoep et al. 2002; Fergusson and Woodward 2002; Breslau et al. 2008, 2011; Leach and Butterworth 2012), and in four studies based on questionnaires for self-report of symptoms (Fletcher 2010; McLeod and Fettes 2007; Duchesne et al. 2008; Morin et al. 2011). 
Fig. 1 Study selection flow chart

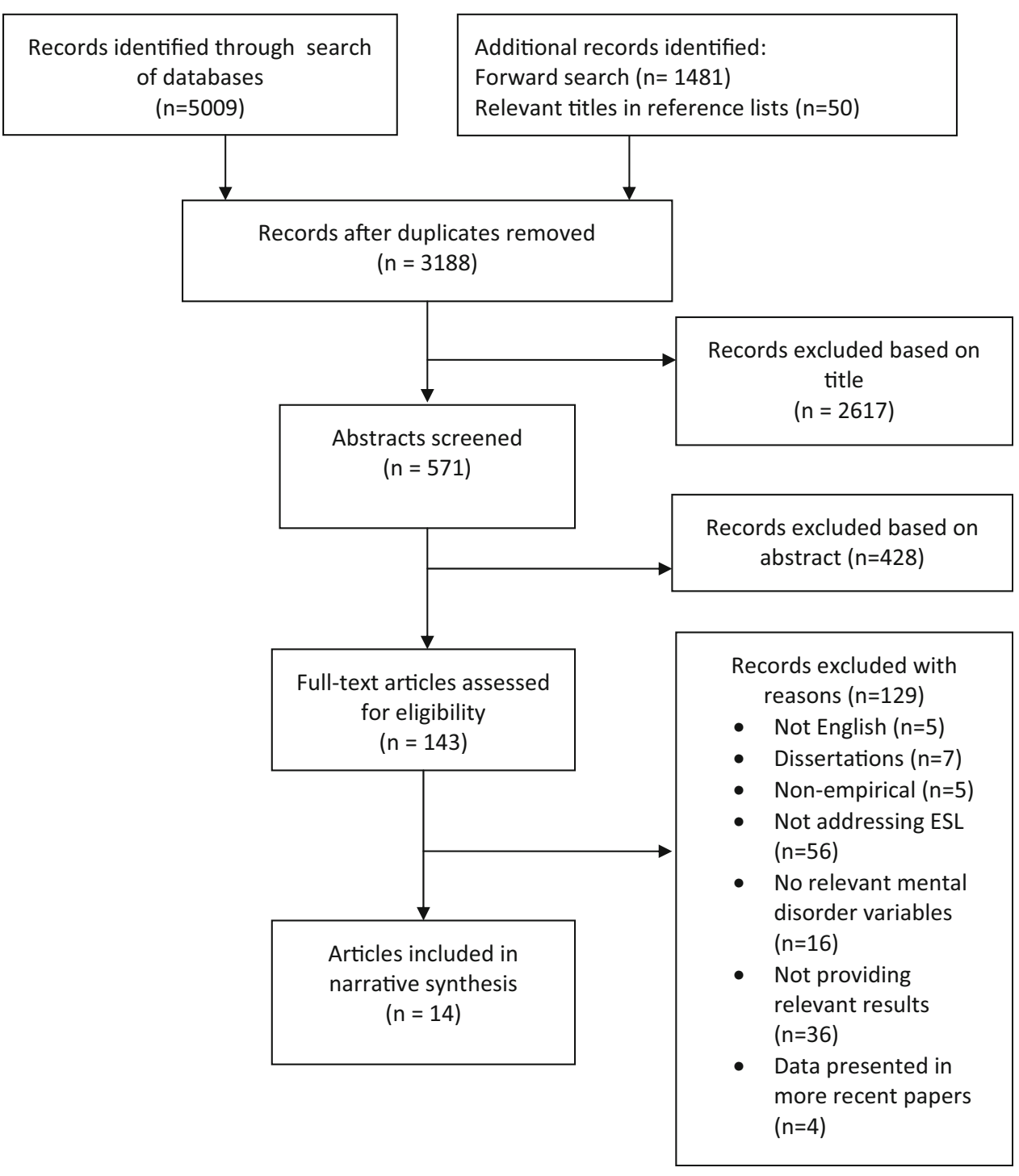

\section{Design}

\section{Prospective Variable-Oriented Studies}

Four prospective variable-oriented studies were identified (Fletcher 2010; Miech et al. 1999; Vander Stoep et al. 2002; Fergusson and Woodward 2002). Two of these studies included measures of both anxiety and depression symptoms (Miech et al. 1999; Vander Stoep et al. 2002) while two reported for depression only (Fletcher 2010; Fergusson and Woodward 2002). Three of the four studies reported increased risk of early school leaving during follow-up among adolescents with internalizing problems (Fletcher 2010; Vander Stoep et al. 2002; Fergusson and Woodward 2002). None of three studies adjusted for comorbidity. Two studies found increased risk for dropping out/early school leaving for adolescents with depression (Fergusson and Woodward 2002; Vander Stoep et al. 2002). Using a continuous scale measuring depressive symptoms, Fletcher (2010) reported one standard deviation in symptom level to be associated with $3.5 \%$ increase in probability for early school leaving. When using a dichotomized variable indicating high levels of depressive symptoms, associations with early school leaving was not found to be statistically significant. One of two studies that included measures of anxiety found it to be a significant risk factor for early school leaving (Vander Stoep et al. 2002). The only prospectivevariable oriented study reporting estimates both with and without adjustment for comorbidity (conduct disorder, anxiety disorder, smoking and alcohol abuse) found that depression was associated with early school leaving before adjustment, but not after (Fergusson and Woodward 2002). 
Table 1 Included studies, reported associations between internalizing problems and early school leaving, and study quality for the included studies

\begin{tabular}{|c|c|c|c|c|c|c|c|}
\hline \multirow[t]{2}{*}{ First author } & \multirow{2}{*}{$\begin{array}{l}\text { Year of } \\
\text { publication }\end{array}$} & \multirow[t]{2}{*}{$\mathrm{N}$} & \multirow[t]{2}{*}{ Country } & \multicolumn{2}{|c|}{ Not comorbidity adjusted } & \multicolumn{2}{|c|}{ Adjusted for comorbidity } \\
\hline & & & & Anxiety & Depression & Anxiety & Depression \\
\hline \multicolumn{8}{|c|}{ Prospective studies } \\
\hline Miech & 1999 & 939 & New Zealand & & & $\mathrm{n} . \mathrm{s}^{\mathrm{d}} / \mathrm{n} . \mathrm{s}^{\mathrm{e}}$ & $\mathrm{n} . \mathrm{s}^{\mathrm{d}} / \mathrm{n} . \mathrm{s}^{\mathrm{e}}$ \\
\hline Fergusson $^{\mathrm{a}}$ & 2002 & 1265 & New Zealand & & $+^{\mathrm{d}}$ & & $\mathrm{n} . \mathrm{s}^{\mathrm{d}}$ \\
\hline Vander Stoep & 2002 & 181 & USA & $+^{\mathrm{d}}$ & $+^{\mathrm{d}}$ & & \\
\hline Fletcher & 2010 & 14,128 & USA & & $\mathrm{n} . \mathrm{s}^{\mathrm{d}} /+^{\mathrm{e}}$ & & \\
\hline \multicolumn{8}{|c|}{ Retrospective studies } \\
\hline Kessler & 1995 & 5877 & USA & $+^{\mathrm{d}}$ & $+^{\mathrm{d}}$ & & \\
\hline Breslau & 2008 & 9282 & USA & $+{ }^{\mathrm{d}}$ & $+{ }^{\mathrm{d}}$ & & \\
\hline $\mathrm{Lee}^{\mathrm{b}}$ & 2009 & 41,688 & International & $+{ }^{\mathrm{d}} / \mathrm{n} \cdot \mathrm{s}^{\mathrm{d}}$ & $+^{\mathrm{d}}$ & & \\
\hline Myer & 2009 & 3370 & South Africa & $\mathrm{n} . \mathrm{s}^{\mathrm{d}}$ & $+^{\mathrm{d}}$ & & \\
\hline Borges & 2011 & 2362 & Mexico & $-^{\mathrm{d}}$ & $\mathrm{n} . \mathrm{s}^{\mathrm{d}}$ & & \\
\hline Breslau & 2011 & 29,662 & USA & $+^{\mathrm{d}}$ & $+^{\mathrm{d}}$ & $\mathrm{n} . \mathrm{s}^{\mathrm{d}}$ & $\mathrm{n} . \mathrm{s}^{\mathrm{d}}$ \\
\hline Leach $^{\mathrm{c}}$ & 2012 & 2055 & Australia & $\mathrm{n} \cdot \mathrm{s}^{\mathrm{d}} /+^{\mathrm{d}}$ & $+^{\mathrm{d}}$ & & \\
\hline \multicolumn{8}{|l|}{ Trajectory studies } \\
\hline Mcleod $^{\mathrm{a}}$ & 2007 & 883 & USA & $+^{\mathrm{e}}$ & & $+{ }^{e}$ & \\
\hline Duchesne & 2008 & 2000 & Canada & $+^{\mathrm{e}}$ & & & \\
\hline Morin & 2011 & 1034 & Canada & $+^{\mathrm{e}}$ & & & \\
\hline
\end{tabular}

+ , Signifies a positive association between internalizing problems and early school leaving; - , signifies a negative association; n.s, signifies nonsignificant associations

${ }^{\text {a }}$ Gender specific results are also reviewed in the results section, but the table only presents overall results

${ }^{\mathrm{b}}$ Results are stratified by income level (high-income countries/low and middle-income countries)

${ }^{c}$ Results stratified by gender (male/female)

d Analysis using diagnosis or high symptom level

e Analyses using symptom level

\section{Retrospective Studies}

Seven studies used retrospective recall of symptoms of internalizing problems, and also age of onset (Kessler et al. 1995; Borges et al. 2011; Lee et al. 2009; Myer et al. 2009; Leach and Butterworth 2012; Breslau et al. 2008, 2011). Five of these used clinical interviews to assess a range of clinical disorders (Kessler et al. 1995; Borges et al. 2011; Myer et al. 2009; Leach and Butterworth 2012; Breslau et al. 2011). All retrospective studies assessed both depressive and anxiety diagnoses.

Six studies reported increased risk for early school leaving among individuals who reported having experienced depression or a mood disorder during adolescence (Lee et al. 2009; Myer et al. 2009; Kessler et al. 1995; Leach and Butterworth 2012; Breslau et al. 2008, 2011), while one study (Borges et al. 2011) reported non-significant associations between mood disorders and early school leaving.

The results were less consistent for anxiety. Three studies reported increased risk for early school leaving for adolescents with one or more types of anxiety disorders
(Kessler et al. 1995; Breslau et al. 2008, 2011). Two studies found anxiety to be associated with increased risk of early school leaving for subgroups of their sample. Leach and Butterworth (2012) reported anxiety disorders to be associated with increased risk of early school leaving for women only. Lee et al. (2009) found that having any anxiety disorder was associated with increased odds for early school leaving in high income countries, while no significant associations were found in low-and middle-income countries. Finally, Borges et al. (2011) found anxiety disorders to be associated with reduced, rather than increased risk for early school leaving.

The only retrospective study adjusting for comorbid externalizing problems (conduct disorder and ADHD) amongst other variables, found no statistically significant association between internalizing problems and early school leaving (Breslau et al. 2011).

\section{Trajectory Studies}

Three of the reviewed studies were trajectory studies (Duchesne et al. 2008; Morin et al. 2011; McLeod and 
Fettes 2007). These were based on repeated measures of internalizing symptoms. By means of various types of mixture modeling, participants were assigned to different groups/classes based upon the similarity in symptom development across time. Thus, the classification of individuals were data-driven, rather than theory driven. Associations were estimated between the different groups and risk for early school leaving. These studies are reviewed separately from the other prospective studies as they report associations between groups of individuals to early school leaving rather than between variables.

Duchesne et al. (2008) used repeated measurements of anxiety symptoms from about 5 years old to about 11 years old and compared the odds of non-completion of high school in each of these groups when participants were 20 years old. This study identified four different trajectory groups differing in their levels of anxiety symptoms across time. The low symptom group comprised about $10 \%$, the moderate symptom group $39 \%$, the high symptom group $41 \%$ and the chronic high symptom group consisted of about $10 \%$ of the sample. The two groups with the highest levels of symptoms were found to be at increased risk for early school leaving compared to the reference group (moderate symptom group).

Morin et al. (2011) identified five trajectory groups based on participants reported anxiety symptoms from about 12 years of age to about 17 years of age. These were the non-anxious (76.8\% of the sample), decreasing anxiety $(2.5 \%)$, school-related anxiety (3.9\%), transition related anxiety $(2.7 \%)$, and the moderate and decreasing anxiety groups $(14 \%)$. The school-related anxiety group who had initially low anxiety levels which increased to peak at the midpoint of the study period and the transition related anxiety group who had initially low levels of symptoms which increased to peak near the end of the study were found to have significantly higher dropout rates than the other groups.

McLeod and Fettes (2007) identified four different trajectories based upon symptoms of internalizing problems from children were 6-8 years old to when they were 18-20 years old and compared the risk for early school leaving relative to the stable low symptoms reference group (67\% of the sample). Relative to the stable low symptoms group, both the childhood high symptoms group (6\% of the sample) and the adolescence high symptoms groups (6\% of the sample) were less likely to complete upper secondary school. After controlling for externalizing problem behaviors, the association between the childhood high symptom group attenuated to statistical non-significance, while the childhood moderate symptom group remained at higher risk for early school leaving relative to the reference group.

\section{Gender Differences}

Nine studies adjusted for gender (Kessler et al. 1995; Borges et al. 2011; Myer et al. 2009; Fletcher 2010; McLeod and Fettes 2007; Duchesne et al. 2008; Vander Stoep et al. 2002; Breslau et al. 2008, 2011), whereas only two of these reported non-significant tests of gender interactions to justify the inclusion of gender as a covariate in the analyses (Kessler et al. 1995; Miech et al. 1999). One additional study tested for gender interaction and reported gender-stratified results despite concluding that differences between genders were not significant (Fergusson and Woodward 2002). In contrast to this, one of the trajectory studies reported reduced risk of early school leaving among girls with increasing anxiety symptom load during adolescence relative to boys (McLeod and Fettes 2007).

\section{Confounding Factors}

Most of the studies included adjustments for socio-economic factors at family level (Kessler et al. 1995; Borges et al. 2011; Lee et al. 2009; McLeod and Fettes 2007; Morin et al. 2011; Miech et al. 1999; Vander Stoep et al. 2002; Fergusson and Woodward 2002; Breslau et al. 2008), but blockwise adjustment with unique sets of covariates limit the comparability and the possibility to draw conclusions regarding the effects of specific covariates. Nevertheless, one study (Fletcher 2010) investigated the role of confounding associated with family and school characteristics by using fixed effects models. The results indicate that non-significant estimates of association attenuated somewhat when including both sibling fixed effects and school level fixed effects. When using a continuous symptom measure for depression, the associations with early school leaving attenuated from a significant OLS estimate of $0.027(p<0.05)$ to a non-significant 0.021 when including sibling fixed effects, and from 0.035 $(p<0.01)$ to $0.032(p<0.01)$ when controlling for school fixed effects.

\section{Comorbid Conditions}

Comorbidity between internalizing disorder and other mental disorders, in particular externalizing disorders, represents an obvious challenge regarding confounding. Only three of the reviewed studies presented estimates both before and after adjusting for comorbid disorders (McLeod and Fettes 2007; Fergusson and Woodward 2002; Breslau et al. 2011). One prospective-variable oriented study (Fergusson and Woodward 2002) and one retrospective study (Breslau et al. 2011) reported non-significant associations between internalizing disorders and early school leaving after adjusting for externalizing problems. 
Nevertheless, these studies also included a range of other covariates in the same analysis limiting the extent to which the attenuation of associations can be attributed to specific variables.

The study of Breslau et al. (2011) included demographic information and childhood adversity as control-variables along with all reported internalizing and externalizing disorders. Their results showed that adjusting for comorbidity attenuated associations for mania from $2.14^{\text {sig }}$ to $1.38^{\text {n.s }}$, for panic from $1.92^{\text {sig }}$ to $1.51^{\text {n.s }}$, for social phobia from $1.40^{\text {sig }}$ to $1.11^{\text {n.s }}$, for specific phobia from $1.24^{\text {sig }}$ to $1.05^{\text {n.s }}$, for PTSD from $1.56^{\text {sig }}$ to $1.11^{\text {n.s }}$, for generalized anxiety disorder from $1.35^{\text {n.s }}$ to $0.78^{\text {n.s }}$, and for depression/ dysthymia from $1.30^{\text {sig }}$ to $.098^{\text {n.s. }}$.

Fergusson and Woodward (2002) showed that associations between major depression during early adolescence and leaving school without educational qualifications attenuated from or $1.8^{\mathrm{sig}}$ to $1.1^{\mathrm{n} . \mathrm{s}}$ when controlling for arrange of covariates including anxiety disorder, conduct disorder and alcohol abuse.

\section{Discussion}

Studies investigating associations between internalizing problems and early school leaving have yielded inconsistent results, but it is unclear whether this inconsistency may be due to differences in study design, statistical models, or which statistical control variables are included. The aim of this systematic review was, therefore, to assess the state of the evidence with regard to whether internalizing problems among children and adolescents constitute a risk factor for early school leaving and whether the inclusion of potential confounding variables influence these results. The extent to which associations vary across gender differences for the association was also investigated.

The main findings of the current review suggests that, regardless of research design, most included studies reported significant associations between internalizing disorders or symptoms thereof and early school leaving. This is an important finding as early school leaving is associated with increased risk for long term unemployment (Kokko et al. 2000), imprisonment (Sum et al. 2009) and increased expenses for the welfare state (Nevala et al. 2011).

Our results indicate that individuals with early onset internalizing disorders are, to some extent, selected into truncated educational careers. To the extent that there is a causal effect of internalizing disorders on early school leaving, this would add to the already strong case for

\footnotetext{
${ }^{1}$ Statistical significance as indicated by $95 \%$ confidence intervals not overlapping with 1 .
}

increased treatment and prevention of these disorders during adolescence. Nevertheless, the confidence in these results should be balanced against the lack of meta-analytic evidence and the limitations discussed below.

The results from the seven retrospective studies are vulnerable for reverse causality, recall bias and selective attrition. Reverse causality may be a problem as the retrospective studies rely on diagnosing mental disorders in the past and age at onset trough diagnostic interviews. Recall bias may influence the results as the internalizing disorders occurring prior to school dropout are reported after individuals have left school, which could result in the confusion of past and present symptoms or conditions. The retrospective studies are also all vulnerable to selective attrition related to the disorders, to the outcome (early school leaving) or to their social consequences. Consequently, institutionalized individuals or those with severe mental illness, who are more likely to have mental illness relative to the general population, are not included in such studies (Kessler et al. 1995). Therefore, we advise caution when interpreting results from retrospective studies.

Confounding is addressed in many of the studies, but with the exception of one study (Leach and Butterworth 2012), all studies used block-wise adjustment for multiple factors, thereby limiting conclusions regarding specific confounders. The study investigating the role of single confounding factors concluded that the estimates of association between depression and early school leaving were relatively robust to unobserved shared sibling characteristics and school characteristics (Fletcher 2010).

Two of the included studies indicate that the relationship between internalizing disorders and early school leaving attenuate to non-significance after controlling for externalizing behavior problems like ADHD (Breslau et al. 2011) and conduct disorder (Fergusson and Woodward 2002). One trajectory-study by McLeod and Fettes (2007) reported that the associations between internalizing trajectories and early school leaving remained largely unchanged when controlling for trajectories of externalizing problem behaviors. The inconsistency in results when comparing the trajectory study (McLeod and Fettes 2007) with the two other studies (Breslau et al. 2011; Fergusson and Woodward 2002) may in part be due to the lack of comparable statistical analyses and different measurement of the independent variables. However, while results are consistent between the studies of Breslau et al. (2011) and Fergusson and Woodward (2002), it is not clear whether these studies provide valid results regarding confounding by externalizing problem behaviors. First, the lack of evidence for a temporal sequence in the time of onset for the different disorders limits our ability to distinguish confounding from mediation. It is, therefore, impossible to determine whether the adjusted estimates are over-adjusted 
or if they represent the true association between internalizing disorders and early school leaving. Second, the statistical adjustment for factors that are correlated with internalizing disorders introduces the possibility of biased estimates due to multicolinearity. Finally, while all estimates of association attenuated and lost their statistical significance when adjusting for externalizing problem behaviors (Breslau et al. 2011; Fergusson and Woodward 2002), some of the more specific disorders such as mania, panic, specific phobia and post-traumatic stress disorder retained odds-ratios of between 1.11 and 1.51, suggesting that studies with larger samples would identify these as statistically significant.

These findings also contrast with the former review by Esch et al. (2014) as they suggest that the association between internalizing disorders and educational attainment to be strongly mediated by co-occurring disruptive behavior problems and by academic achievement. While it is indeed possible that internalizing problems may lead to disruptive behavior problems and poor academic achievement which in turn may lead to low educational attainment or early school leaving, none of the cited references (Miech et al. 1999; Breslau et al. 2011) include appropriate mediation analyses which would allow for such a temporal sequence to be tested. We therefore consider the cause for the attenuation of associations after adjustment for externalizing problem behaviors to be unknown. In sum, the lack of consistency in results and general limitations in the reviewed studies suggest that the evidence is inconclusive with regard to potential confounding or mediation of externalizing problem behaviors.

Comorbidity is not only relevant due to potential confounding, but also with regard to the risk associated with having multiple disorders, or specific combinations of disorders. Four studies provided results indicating that the risk of early school leaving increased with the number of disorders (Kessler et al. 1995; Borges et al. 2011; Lee et al. 2009; Breslau et al. 2008). However, these results were based upon non-specific comorbidity and are not necessarily relevant for the current investigation. Only one study addressed specific types of comorbidity and reported that the odds for early school leaving were not different for adolescents with both an internalizing disorder and conduct disorder or ADHD, relative to adolescents with only conduct disorders or ADHD (Breslau et al. 2011). In the trajectory studies, it is unclear to what extent the analysis is capable of separating internalizing from externalizing problems.

Attrition is an obvious source of bias in the majority of studies not relying on registry outcomes in both prospective and trajectory studies. Obviously, adolescents who have dropped out of school are less likely to participate in follow-ups and mental disorder in itself has been shown to be related to non-participation (Bergman et al. 2010). In sum, this is highly problematic as attrition during follow-up and participation bias related to mental disorder prior to dropout may lead to biased estimates.

Our findings suggest that gender differences in associations between internalizing problems and early school leaving have not been a prioritized area for most of the reviewed studies. Only two studies reported stratified analyses for gender, indicating a stronger association between internalizing problems and early school leaving in girls than in boys (Fergusson and Woodward 2002; Leach and Butterworth 2012). However, these gender differences were not supported by statistical analyses as three (Kessler et al. 1995; Miech et al. 1999; Fergusson and Woodward 2002) out of four studies testing for gender interactions conclude that associations are not different for boys and girls. The final study concluded that girls with increasing levels of anxiety throughout adolescence are at decreased risk of early school leaving relative to boys (McLeod and Fettes 2007). This suggests that educational outcomes for adolescents with internalizing problems appear to be equally severe for boys and girls.

Esch and colleagues conclude differently from our interpretation of the literature as they suggest that there is some evidence for effect modifications by sex (Esch et al. 2014). Two studies are cited in support of the argument that consequences of internalizing disorders are more severe for females compared to males (Kessler et al. 1995; Fletcher 2008). Fletcher (2008) do indeed present significant associations between depression and dropout for girls, but not for boys. While these differences may possibly be significantly different, no statistical tests are presented to verify this assumption. The study of Kessler et al. (1995) actually report that there were no significant gender differences, and the presented regression models therefore constrain the effects of disorders to be equal across genders. This study does, however, report a higher prevalence of prior psychiatric disorders among individuals who do not complete high school, but these gender differences are due to prevalence differences and not due to gender modification of the association between disorders and early school leaving. In sum, we argue that while evidence is not overwhelmingly consistent, there is little support for the gender difference in associations between internalizing disorders and educational attainment as presented by Esch et al. (2014).

The differences in statistical approaches used provided a major challenge for the comparison between studies. First, all studies controlled for different set of covariates, and only one study presented true crude associations (not adjusted for any other variable). Hence, comparability of results across studies was precluded as differing sets of covariates will produce non-comparable estimates (Mood 
2010). Second, studies differed in how they reported the outcome variable as some studies reported odds of dropping out at different grade levels (Kessler et al. 1995; Borges et al. 2011; Breslau et al. 2008; Leach and Butterworth 2012). Although this stratification provides more nuanced information about risk factors for leaving school at different stages of their education, it reduces statistical power and comparability with studies that have investigated risk for not completing year 12 only. Third, different types of statistical analysis made comparison across studies difficult. While a majority of the included studies reported results from different types of binary regression models where completing 12 years of school or not was regressed upon symptom level or diagnosis (Kessler et al. 1995; Borges et al. 2011; Fletcher 2010; Miech et al. 1999; Vander Stoep et al. 2002; Fergusson and Woodward 2002; Breslau et al. 2008; Leach and Butterworth 2012), three of the included studies (McLeod and Fettes 2007; Duchesne et al. 2008; Morin et al. 2011) used individual oriented analyses where results were difficult to compare as the reference groups used varied across studies. Nevertheless, these studies concur with the main findings of the paper in that individuals with high symptom levels are less likely to complete upper secondary school relative to individuals with lower symptom levels.

Finally, the lack of long-term follow-up of the educational status is a limitation when aiming to address early school leaving. All of the included longitudinal studies assessed educational status when participants were between 18 and 22 (Fletcher 2010; McLeod and Fettes 2007; Duchesne et al. 2008; Morin et al. 2011; Miech et al. 1999; Vander Stoep et al. 2002; Fergusson and Woodward 2002) and only one study (Duchesne et al. 2008) reported the proportion of individuals who were still in school at the time dropout was assessed. In this study over $70 \%$ of those registered as dropouts were still in school. International reports also show that at least $10 \%$ of those who complete upper secondary school in Denmark, Finland, Iceland, New Zealand, Norway and Portugal are 25 or older (OECD 2011). Consequently, this indicates that some of the reviewed studies assess risk factors for failing to follow the normative progression through the educational stages rather than early school leaving.

There are some limitations to our study. First, we did no attempts to include unpublished data or grey literature, which if included could reduce publication bias. Second, the systematic review conducted a search only on studies published in English, and only three studies were from nonEnglish speaking countries (Borges et al. 2011; Lee et al. 2009; Myer et al. 2009). This limitation may be relevant as the effect of internalizing disorders on early school leaving is likely to interact with context and opportunity and may explain the somewhat unexpected "protective" effect of anxiety as regards early school leaving in a sample from Mexico (Borges et al. 2011). A third limitation is the lack of a standardized risk of bias assessment. While this is generally recommended in systematic reviews (Higgins and Green 2008), we found that the established risk of bias assessment scales were of limited benefit when reviewing observational studies with substantial methodological heterogeneity. Nevertheless we have addressed three out of the five key areas of bias as pointed out by the Cochrane Handbook for Systematic Reviews of Interventions (Higgins 2014). Selection bias is discussed in light of the retrospective studies, the omitted variable discussion addresses potential performance bias, and attrition bias is discussed as relevant for the prospective studies. Detection bias was considered irrelevant for the included studies as outcomes were assessed in similar ways across all participants. Finally we also acknowledge that reporting bias may be a relevant source of bias due to publication bias or selective outcome reporting.

Future studies should aim to overcome the reviewed shortcomings in the literature by applying prospective and historical cohort designs, employ validated screening instruments, minimize the risk of attrition by employing registry information for outcomes, include the most relevant confounders at baseline including social background factors at family and macro level, and-probably most importantly - analyze and report how comorbid disorders including externalizing disorders including ADHD and conduct disorder, influence the associations. Studies with long term follow-up are also needed in order to distinguish early school leaving from delayed academic progression. Reporting needs to include both crude and adjusted effects, and covariates attenuating the association of interest should be highlighted.

\section{Conclusions}

The majority of studies suggest that adolescents with anxiety and depression are less likely to complete upper secondary school. There is limited, but relatively consistent evidence suggesting that this association is stable across gender. Externalizing problem behaviors and ADHD appear to attenuate the association between internalizing problems and early school leaving, but it is not clear whether this is due to confounding or mediation. Hence, our results indicate that adolescents with anxiety or depression indeed are at higher risk for early school leaving, but that the causal mechanism behind this association is unclear.

Acknowledgments The study is funded by the Research Council of Norway. We acknowledge the authors of all included articles, the library of the National Institute of Public Health, and the Norwegian 
Knowledge Centre for the Health Services for providing advice with regard to search methodology and systematic reviewing. We are also grateful for the comments and help from the authors of several of the included articles.

Authors' Contributions O. M. conducted the systematic search and wrote the manuscript. O. M. and W. N. screened and reviewed all studies. W. N., M. E., A. R. and A. M. provided O. M. with feedback during all stages of the process and wrote some sections of the manuscript. All authors have read and approved of the final manuscript.

\section{Compliance with Ethical Standards}

Conflict of interest The authors declare that they no conflict of interest.

\section{Appendix}

The search strings used:

Internalizing disorders: anxi* OR depress* OR mental health* OR common mental disorder OR cmd OR mental distress OR internaliz* OR internalis* OR "emotional problem*" OR "emotional distress*" OR "emotional disorder" OR "mood disorder*".

Early school leaving: ((education* OR school OR academic) AND (termination OR dropout OR "drop* out" OR "early leaving" OR failure OR *achievement OR *completi* OR attainment OR graduati*)).

\section{References}

Bergman, P., Ahlberg, G., Forsell, Y., \& Lundberg, I. (2010). Nonparticipation in the second wave of the PART study on mental disorder and its effects on risk estimates. International Journal of Social Psychiatry, 56(2), 119-132.

Bjelland, I., Krokstad, S., Mykletun, A., Dahl, A. A., Tell, G. S., \& Tambs, K. (2008). Does a higher educational level protect against anxiety and depression? The HUNT study. Social Science and Medicine, 66(6), 1334-1345.

Borges, G., Mora-Icaza, M. E. M., Benjet, C., Lee, S., Lane, M., \& Breslau, J. (2011). Influence of mental disorders on school dropout in Mexico. Revista Panamericana de Salud Pública, 30(5), 477-483.

Breslau, J., Lane, M., Sampson, N., \& Kessler, R. C. (2008). Mental disorders and subsequent educational attainment in a US national sample. Journal of Psychiatric Research, 42(9), 708-716.

Breslau, J., Miller, E., Joanie Chung, W.-J., \& Schweitzer, J. B. (2011). Childhood and adolescent onset psychiatric disorders, substance use, and failure to graduate high school on time. Journal of Psychiatric Research, 45(3), 295-301.

Duchesne, S., Vitaro, F., Larose, S., \& Tremblay, R. E. (2008). Trajectories of anxiety during elementary-school years and the prediction of high school noncompletion. Journal of Youth and Adolescence, 37(9), 1134-1146.

Esch, P., Bocquet, V., Pull, C., Couffignal, S., Lehnert, T., Graas, M., et al. (2014). The downward spiral of mental disorders and educational attainment: A systematic review on early school leaving. BMC psychiatry, 14(1), 237.

Fergusson, D. M., \& Woodward, L. J. (2002). Mental health, educational, and social role outcomes of adolescents with depression. Archives of General Psychiatry, 59(3), 225-231.

Fletcher, J. M. (2008). Adolescent depression: Diagnosis, treatment, and educational attainment. Health Economics, 17(11), 1215-1235.

Fletcher, J. M. (2010). Adolescent depression and educational attainment: Results using sibling fixed effects. Health Economics, 19(7), 855-871.

Fryers, T., Melzer, D., \& Jenkins, R. (2003). Social inequalities and the common mental disorders. Social Psychiatry and Psychiatric Epidemiology, 38(5), 229-237.

Higgins, J. (2014). Green S. Cochrane handbook for systematic reviews of interventions Version 5.1. 0 [updated March 2011]. The Cochrane Collaboration, 2011. www.cochrane-handbook. org.

Higgins, J. P., \& Green, S. (2008). Cochrane handbook for systematic reviews of interventions (Vol. 5). London: Wiley.

Jayakody, R., Danziger, S., \& Kessler, R. C. (1998). Early-onset psychiatric disorders and male socioeconomic status. Social Science Research, 27(4), 371-387.

Kessler, R. C., Berglund, P., Demler, O., Jin, R., Merikangas, K. R., \& Walters, E. E. (2005a). Lifetime prevalence and age-of-onset distributions of DSM-IV disorders in the National Comorbidity Survey Replication. Archives of General Psychiatry, 62(6), 593-602.

Kessler, R. C., Chiu, W. T., Demler, O., \& Walters, E. E. (2005b) Prevalence, severity, and comorbidity of 12-month DSM-IV disorders in the National Comorbidity Survey Replication. Archives of General Psychiatry, 62(6), 617-627.

Kessler, R. C., Foster, C. L., Saunders, W. B., \& Stang, P. E. (1995). Social consequences of psychiatric disorders, I: Educational attainment. American Journal of Psychiatry, 152(7), 1026-1032.

Kessler, R. C., McGonagle, K. A., Swartz, M., Blazer, D. G., \& Nelson, C. B. (1993). Sex and depression in the National Comorbidity Survey I: Lifetime prevalence, chronicity and recurrence. Journal of Affective Disorders, 29(2), 85-96.

Knudsen, A., Harvey, S., Mykletun, A., \& Øverland, S. (2013). Common mental disorders and long-term sickness absence in a general working population. The Hordaland Health Study. Acta Psychiatrica Scandinavica, 127(4), 287-297.

Kokko, K., Pulkkinen, L., \& Puustinen, M. (2000). Selection into long-term unemployment and its psychological consequences. International Journal of Behavioral Development, 24(3), 310-320.

Leach, L. S., \& Butterworth, P. (2012). The effect of early onset common mental disorders on educational attainment in Australia. Psychiatry Research, 199(1), 51-57.

Lee, J. M., Jr, Contreras, F., McGuire, K. M., Flores-Ragade, A., Rawls, A., Edwards, K., et al. (2011). The college completion agenda: 2011 progress report. New York, NY: College Board Advocacy and Policy Center.

Lee, S., Tsang, A., Breslau, J., Aguilar-Gaxiola, S., Angermeyer, M., Borges, G., et al. (2009). Mental disorders and termination of education in high-income and low-and middle-income countries: Epidemiological study. The British Journal of Psychiatry, 194(5), 411-417.

Macleod, J., Oakes, R., Copello, A., Crome, I., Egger, M., Hickman, M., et al. (2004). Psychological and social sequelae of cannabis and other illicit drug use by young people: A systematic review of longitudinal, general population studies. The Lancet, 363(9421), 1579-1588.

McLeod, J. D., \& Fettes, D. L. (2007). Trajectories of failure: The educational careers of children with mental health problems. American journal of Sociology, 113(3), 653. 
McLeod, J. D., \& Kaiser, K. (2004). Childhood emotional and behavioral problems and educational attainment. American Sociological Review, 69(5), 636-658.

Miech, R. A., Caspi, A., Moffitt, T. E., Wright, B. R. E., \& Silva, P. A. (1999). Low socioeconomic status and mental disorders: A longitudinal study of selection and causation during young adulthood 1. American Journal of Sociology, 104(4), 1096-1131.

Moher, D., Liberati, A., Tetzlaff, J., \& Altman, D. G. (2009). Preferred reporting items for systematic reviews and metaanalyses: The PRISMA statement. Annals of Internal Medicine, 151(4), 264-269.

Mood, C. (2010). Logistic regression: Why we cannot do what we think we can do, and what we can do about it. European Sociological Review, 26(1), 67-82.

Morin, A. J., Maïano, C., Nagengast, B., Marsh, H. W., Morizot, J., \& Janosz, M. (2011). General growth mixture analysis of adolescents' developmental trajectories of anxiety: The impact of untested invariance assumptions on substantive interpretations. Structural Equation Modeling: A Multidisciplinary Journal, 18(4), 613-648.

Myer, L., Stein, D., Jackson, P., Herman, A., Seedat, S., \& Williams, D. (2009). Impact of common mental disorders during childhood and adolescence on secondary school completion. South African Medical Journal, 99(5), 354-356.

Mykletun, A., Bjerkeset, O., Dewey, M., Prince, M., Overland, S., \& Stewart, R. (2007). Anxiety, depression, and cause-specific mortality: The HUNT study. Psychosomatic Medicine, 69(4), 323-331.

Mykletun, A., Bjerkeset, O., Øverland, S., Prince, M., Dewey, M., \& Stewart, R. (2009). Levels of anxiety and depression as predictors of mortality: The HUNT study. The British Journal of Psychiatry, 195(2), 118-125.

Mykletun, A., Overland, S., Dahl, A. A., Krokstad, S., Bjerkeset, O., Glozier, N., et al. (2006). A population-based cohort study of the effect of common mental disorders on disability pension awards. The American journal of psychiatry, 163(8), 1412-1418.

Needham, B. L. (2009). Adolescent depressive symptomatology and young adult educational attainment: An examination of gender differences. Journal of Adolescent Health, 45(2), 179-186.
Nevala, A.-M., Hawley, J., Stokes, D., Slater, K., Souto-Otero, M., Santos, R., et al. (2011). Reducing early school leaving in the EU. Directorate General for Internal Policies, European Parliament.

OECD. (2011). Education at a Glance 2011: OECD Indicators, OECD Publishing. doi:10.1787/eag-2011-en.

Sum, A., Khatiwada, I., \& McLaughlin, J. (2009). The consequences of dropping out of high school: Joblessness and jailing for high school dropouts and the high cost for taxpayers.

van der Kolk, A., Bouwmans, C., van der Gaag, R.-J., Buitelaar, J., Hakkaart-van Roijen, L., \& van Agthoven, M. (2011). A systematic review of the burden of illness (BOI) of attention deficit hyperactivity disorder (ADHD) regarding educational performance and work. In Journal of mental health policy and economics, 2011 (Vol. 14, pp. S36-S37). International Center of Mental Health Policy and Economics-ICMPE via Daniele Crespi 17, Milano, Italy.

Vander Stoep, A., Weiss, N. S., Kuo, E. S., Cheney, D., \& Cohen, P. (2003). What proportion of failure to complete secondary school in the US population is attributable to adolescent psychiatric disorder? The Journal of Behavioral Health Services and Research, 30(1), 119-124.

Vander Stoep, A., Weiss, N. S., McKnight, B., Beresford, S. A., \& Cohen, P. (2002). Which measure of adolescent psychiatric disorder-Diagnosis, number of symptoms, or adaptive functioning-Best predicts adverse young adult outcomes? Journal of Epidemiology and Community Health, 56(1), 56-65.

Wang, J. L., Schmitz, N., \& Dewa, C. (2010). Socioeconomic status and the risk of major depression: The Canadian National Population Health Survey. Journal of Epidemiology and Community Health, 64(5), 447-452.

Whiteford, H. A., Degenhardt, L., Rehm, J., Baxter, A. J., Ferrari, A. J., Erskine, H. E., et al. (2013). Global burden of disease attributable to mental and substance use disorders: Findings from the Global Burden of Disease Study 2010. The Lancet, 382(9904), 1575-1586. 